\title{
TEXTURE IN Nd-Fe-B ALLOYS PRODUCED BY RAPID SOLIDIFICATION
}

\author{
J.A. SZPUNAR*, P. BLANDFORD*, Z.ALTOUNIAN**, J.STROM- \\ OLSEN $^{* *}$ and A. ZALUSKA** \\ **Department of Physics, *Department of Metallurgical Engineering, \\ McGill University, 3450 University St., Montreal, Quebec, H3A 2A7, \\ Canada.
}

The texture and microstructure of melt-spun ribbons was studied using X-ray diffraction, pole figures and SEM. At constant wheel speed, the nozzle diameter affects the quenching conditions. The observed changes were responsible for a drastic variation of the strength of texture through the thickness of the specimens, and also for significant differences in the microstructure. We have demonstrated that the observed differences in the texture are related to the grain size and indicate that there is a relationship between the growth rate of a grain and its orientation.

\section{THE ROLE OF TEXTURE IN Nd-Fe-B MAGNETS}

A high energy product, $(\mathrm{BH})_{\max }$, characterizes hard magnetic materials. A large remanent induction and a large coercive force are conditioned by a suitable microstructure and also depend on the fundamental physical properties of the magnetic compound.

The properties of the $\mathrm{Nd}_{2} \mathrm{Fe}_{14} \mathrm{~B}$ magnetic phase are very attractive. The magnetic moment per atom is high which assures a high value of magnetization. The tetragonal magnetic phase has a high uniaxial anisotropy with an easy magnetic direction along the c-axis. Less advantageous is the Curie temperature (588K) 1 which is a limiting factor for applications of the magnet even at moderately high temperatures.

The high coercive force and the high remanence can be achieved by a suitable control of the microstructure and texture. In order to have a high coercive force, the magnet should consist of small grains representing single domain particles. To obtain the high remanent induction, these particles must exhibit a preferred axis of magnetization and should be aligned so that the magnetic specimen is highly anisotropic.

This alignment of particles, or so called texture, is responsible for the significant increase of the energy product $(\mathrm{BH})_{\max }$, i.e. from $10 \mathrm{MGOe}$ to 40 MGOe. Making a strongly textured magnet is therefore a considerable 
challenge for processing technology. The alloy is usually prepared by arc melting under an argon atmosphere from commercial purity constituents. $\mathrm{HfB}_{2}$ is often added 2 to introduce fine dispersoids as a means of grain refinement. The melt spinning technique is then used to produce narrow (10 $\mu \mathrm{m}$ thick, $100 \mu \mathrm{m}$ wide), short, amorphous or crystalline filaments. These filaments, often isotropic, are then powdered. Such material is then hot extruded, and during this process texture is introduced. The type and strength of texture depends on the distribution of the normal stresses and strains during extrusion and also on the extrusion temperature

Applications of Nd-Fe-B magnets has stimulated various types of research. In this paper we describe investigations of the relationship between the quench rate and crystallization and texturing of alloys produced by rapid solidification.

\section{EXPERIMENTAL DETAILS}

Specimens were prepared by melt spinning of a Nd-Fe-B alloy. The purity of the raw materials was $99.9 \%$ for $\mathrm{Nd}$ and $\mathrm{B}$ and $99.98 \%$ for Fe. Appropriate amounts of these elements were induction melted at least five times in a water cooled copper boat in order to achieve a high homogeneity. The alloy buttons were melt-spun in an oxygen-free argon atmosphere using a steel wheel rotating with a constant surface velocity of $20 \mathrm{~m} / \mathrm{s}$. Three different nozzle diameters were used, $0.8 \mathrm{~mm}, 0.72 \mathrm{~mm}$ and $0.65 \mathrm{~mm}$.

All X-ray diffraction measurements were made using a Rigaku rotating anode generator equipped with a powder diffractometer and computerized diffraction data base. Texture measurements were made using a Siemens D-500 unit equipped with a Eulerian cradle for the pole figure measurements. The pole figures were measured up to a maximum tilting angle of 80 degrees. Co $\mathrm{Ka}$ radiation was used in all texture experiments.

Scanning electron microscopy was used to observe the variation in the microstructure through the flake thickness.

\section{RESULTS AND DISCUSSION}

The crystallinity, texture and magnetic properties of the ribbons depends critically on the quench rate. Normally, the samples are produced by ejecting molten alloy onto the edge of a rotating wheel. The quench rate is critically dependant on the wheel speed and diameter of the nozzle, but also depends on several other parameters, for instance, the nozzle ejection pressure, the distance between the nozzle and the wheel surface, the type of protective atmosphere and its pressure, and the type of wheel material and its surface condition.

Wheel speed is normally related to the quench rate and therefore, can change the proportion between the volume of the crystalline and the amorphous phases. A low speed, of the order of $10 \mathrm{~m} / \mathrm{s}^{1}$, gives the crystalline 
phase $\mathrm{Nd}_{2} \mathrm{Fe}_{14} \mathrm{~B}$ only. A high speed, of about $60 \mathrm{~m} / \mathrm{s}$, gives specimens which are almost completely amorphous.

All our specimens were prepared with the same wheel speed of $20 \mathrm{~m} / \mathrm{s}$ and statistically could contain about $10 \%$ of the amorphous phase. They were prepared using different nozzle diameters and therefore the crystallization and texturing process has become much more complex.

A first indication of this complexity can be found by analyzing a series of X-ray diffraction patterns which were obtained from the free side of the ribbons. Similarly, diffraction patterns were obtained for the wheel sides of the flakes. The results obtained indicate that for a nozzle diameter of $\mathrm{D}=0.65 \mathrm{~mm}$, texture is observed at the free side of the ribbon, while for a nozzle diameter of $D=0.72 \mathrm{~mm}$, the texture is seen on the wheel side. For a larger nozzle diameter of $\mathrm{D}=0.8 \mathrm{~mm}$, the texture on the wheel side of the ribbon becomes even stronger. This texture information was derived from the intensities of five diffraction maxima. There is only one type of texture present, this having the c-axis of the crystallites perpendicular to the surface of the flake.

A summary of the X-ray diffraction analysis is given in Figure 1, in the form of a diagram illustrating the strength of texture in relation to the nozzle diameter, for both the wheel and the free sides of the ribbons. The texture transition from the free side $(D=0.65 \mathrm{~mm})$ to the wheel side $(D=0.8$ $\mathrm{mm}$ ) is an interesting phenomena which we will not be in a position to explain fully at present. However, this observation clearly indicates that the quench rate is not the same at various depths throughout the specimen and the texture might develop as a result of a selective growth process.

More quantitative, pole figure measurements of the same materials fully support the presented X-ray diffraction measurements. Pole figures for the $\{006\}$ reflection measured using Co $\mathrm{Ka}$ radiation were obtained for the free side of the specimens and are presented in Figures 2a,b and c. Pole figure measurements were also done for the middle layer of the flakes (half way through the flake thickness). The measurements of the pole figures on semi-crystalline $\mathrm{Nd}-\mathrm{Fe}-\mathrm{B}$ are extremely difficult and offers many experimental challenges. The flakes were small and fragmented and their surfaces were bent. In addition, the background in the pole figure measurements was high, and the peaks were overlapping because fine detector collimation is not recommended for pole figure measurements. In order to obtain the pole figures presented, the background corrections and defocussing corrections were made for the entire pole figure and a special method of deconvolution was used to extract the data

The pole figures given in Figure 2 show that on the free surface of the specimens, $(D=0.65 \mathrm{~mm})$ the texture is strong, having a maximum of 4.6 in random units. For a nozzle diameter of $D=0.72 \mathrm{~mm}$ and $\mathrm{D}=0.8 \mathrm{~mm}$, the texture is not as strong and is about 2.2 units. This result confirms our previous observation of the texture transition from the free side $(D=0.65$ $\mathrm{mm}$ ) to the wheel side $(\mathrm{D}=0.72 \mathrm{~mm})$. By measuring the pole figures at halfflake thickness, we demonstrated that there is no texture there. 


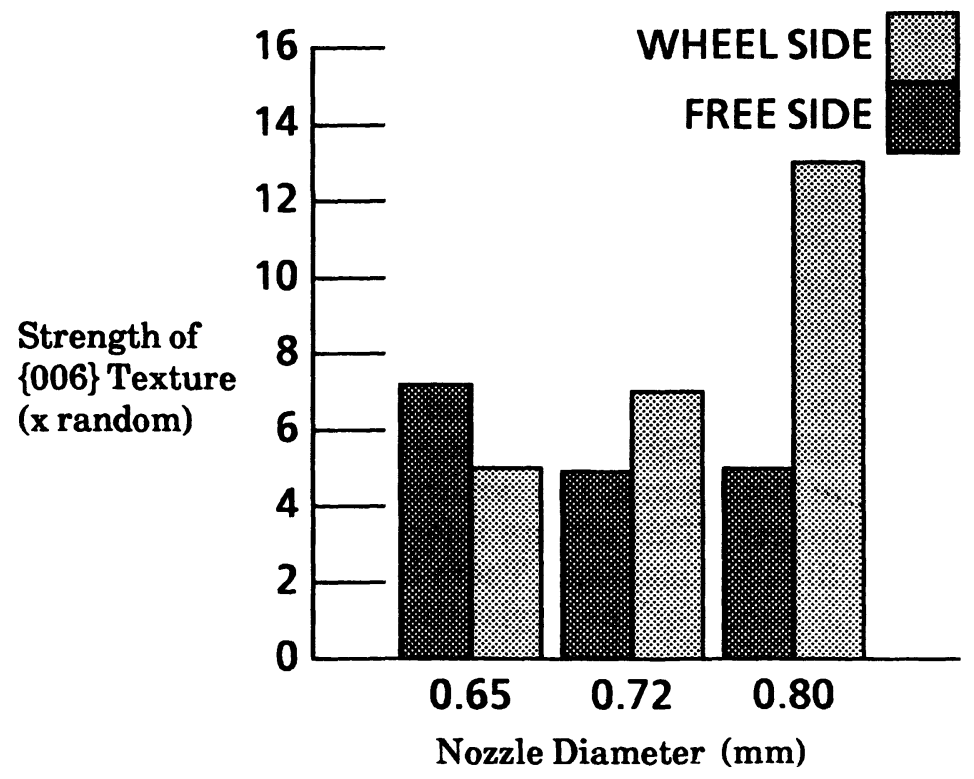

Figure 1:Summary of diffraction results obtained for different nozzle diameters.

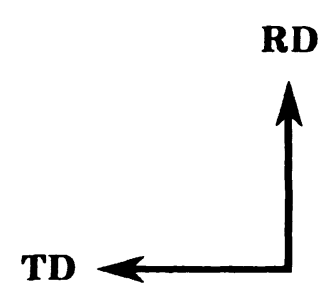

Contours: $1,2,3,4,5$

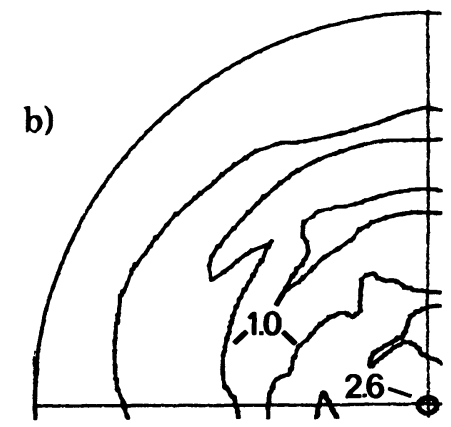

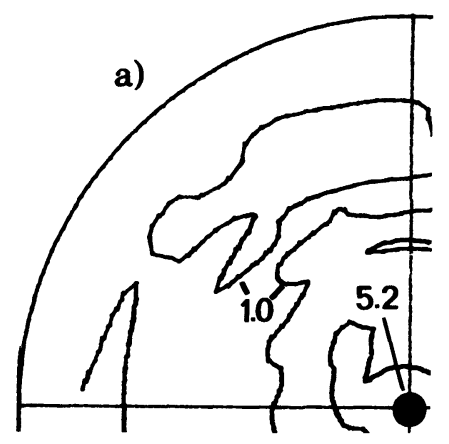

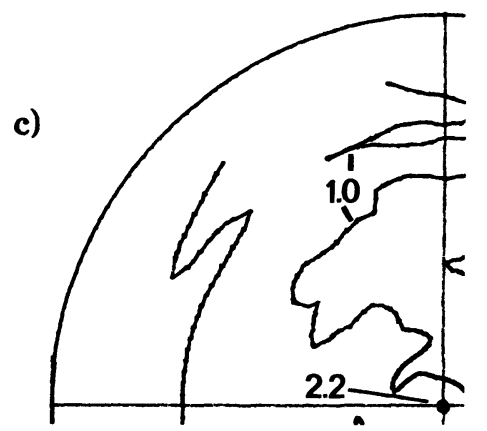

Figure 2: $\{006\}$ pole figures for the free side of flakes made with nozzle diameters a) $\mathrm{D}=0.65 \mathrm{~mm}, \mathrm{~b}) \mathrm{D}=0.72 \mathrm{~mm}$ and c) $\mathrm{D}=0.80 \mathrm{~mm}$. 
We will now present the results of the microstructural observations done on the plane perpendicular to the surface of the flakes. These observations were done using an SEM (Joel T 300 microscope operating at 20 $\mathrm{kV}$ ) and have been reported previously3. The micrograph of the smaller nozzle diameter specimen $(D=0.65 \mathrm{~mm})$, Figure $3 \mathrm{a}$, shows that the grain size is largest (about $30 \mathrm{~nm}$ ) on the free side of the ribbon and becomes smaller when we move towards the wheel side. The cooling of the melt-spun alloy is obviously most efficient near the surface which makes contact with the wheel.

Figure 3b shows the fractured surface of the ribbon quenched with nozzle diameter of $\mathrm{D}=0.8 \mathrm{~mm}$. Here, on the wheel side of the ribbon, the texture is strong and the grain size as estimated by Zaluska, et al ${ }^{3}$, is between 40 and $100 \mathrm{~nm}$. The decrease of the strength of texture on the free side is associated with the large grain size of about $6 \mu \mathrm{m}$.

The microstructural observations presented here clearly point out that there might exist a correlation between the grain orientation and the grain growth rate; grains about $30 \mathrm{~nm}$ in size are textured, while grains larger than about $0.5 \mu \mathrm{m}$ are not. It is also possible to speculate that the change in quench rate existing at various depths in the specimen and the quench rate anisotropy has an impact on the texture development on both sides of the ribbon. Further studies on the texturing of melt-spun Nd-Fe-B alloys are in progress.

\section{CONCLUSIONS}

The texture observed in melt-spun Nd-Fe-B alloys change through the thickness of the flake, and its type and strength can be different on both sides of the ribbon. We have demonstrated here that, for the specimens produced at a constant wheel speed of $20 \mathrm{~m} / \mathrm{s}$, the nozzle diameter is responsible for drastic changes in the texture and the microstructure. In particular, the texture disappears from the free side of the flake and appears on the wheel side. We have demonstrated that the aforementioned texture transition is related to the grain size, and most likely can be explained by a relationship between grain growth rate and grain orientation.

ACKNOWLEDGEMENTS: This research was supported by the National Science and Engineering Research Council of Canada.

\section{REFERENCES:}

1 Y. Xu, W.B. Miur and Z. Altounian, J. Magn. Magn. Mat., 82, 43 (1989).

2 C.J. Yang, Textures and Microstructures, 11, 143 (1989).

3 A.Zaluska, Y. Xu, Z. Altounian and J. Strom-Olsen, Proc. of the Conference on Rapidly Quenched Metals 7, Stockholm (1990). 

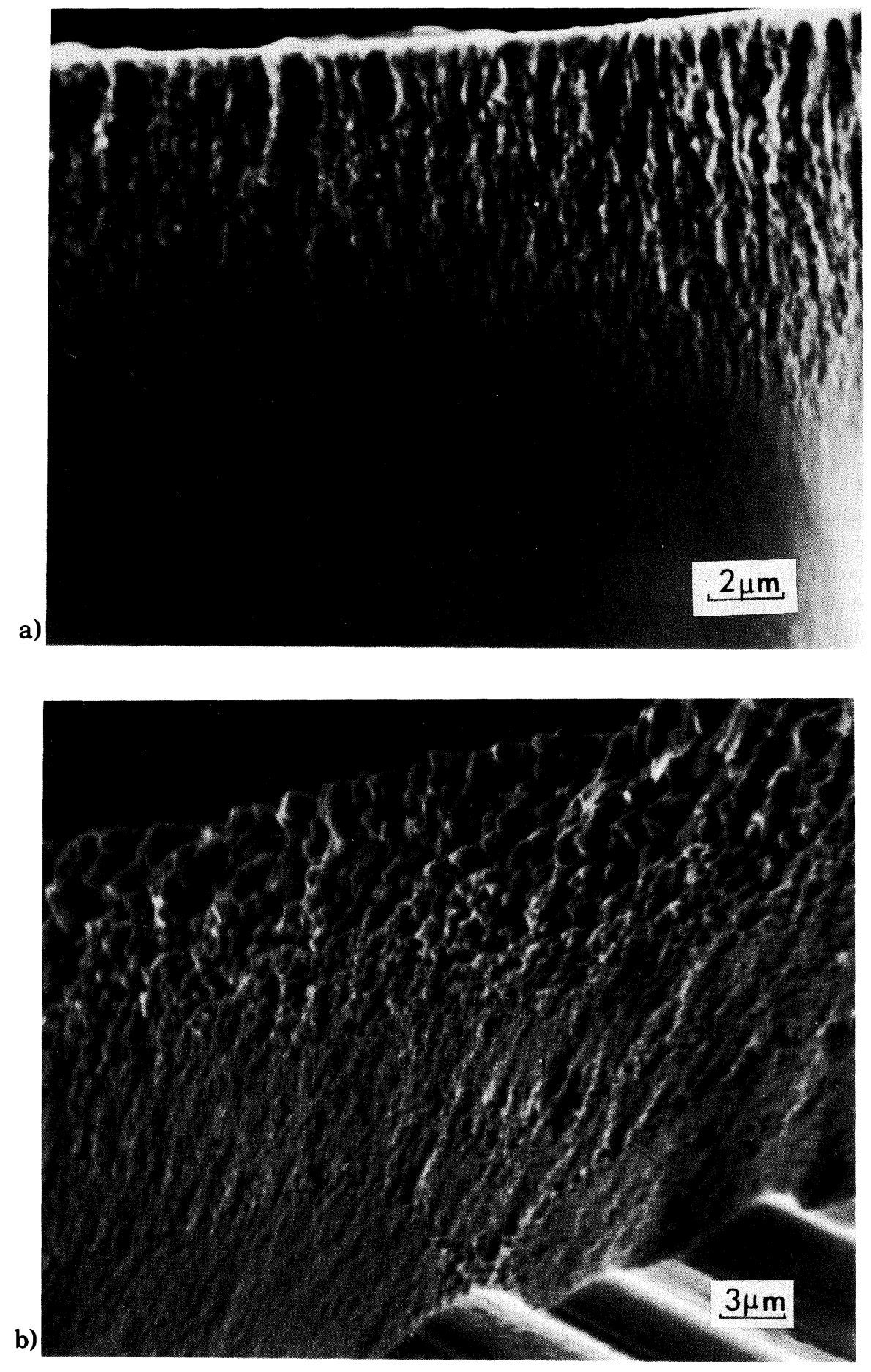

Figure 3: SEM micrographs (at $20 \mathrm{kV}$ ) of the cross sections of flakes spun with nozzle diameters a) $\mathrm{D}=0.65 \mathrm{~mm}$ and b) $\mathrm{D}=0.80 \mathrm{~mm}$. 